\section{Batida de pé, différenciations et mélanges musico-chorégraphiques}

Por Jean-Michel Beaudet

Université Paris Ouest - Nanterre La Défense

(jeanmichelbeaudet@yahoo.fr)

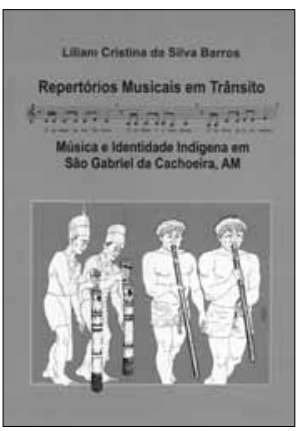

BARROS, Líliam.

Repertórios musicais

em trânsito. Música

e identidade indígena

em São Gabriel da

Cachoeira, AM.

Belém: EDUFPA,

2008. 290 p. il. ISBN

9788524704819

Au cours d'un long entretien, une brincante d'une association a précisé :

(...) As músicas são compostas pelos músicos da agremiação. Os músicos são pagos. $\bigcirc$ ritmo é de 'batida do pé', diferentemente do que acontece em Barcelos, que é 'bailado'. A música é regional, parecida com os passos do cariço. Essa diferença aparece, por exemplo, nas músicas da agremiação Baré, que é de Parintins e é música de Boi. A 'batida de pé' é só com a perna direita (...) (p. 92).

Contacts, mélanges et distinctions entre différentes pratiques musico-chorégraphiques, multiplicité et croisements entre les différentes catégorisations locales appliquées à ces répertoires et à ces pratiques, ce livre nous offre une ethnologie très intéressante de la vie musicale actuelle à São Gabriel da Cachoeira (AM). Ainsi, dans l'extrait cité, une actrice de ces évènements désigne en quelques mots des processus créatifs, des caractéristiques économiques, des distinctions socio-spatiales, des catégories appliquées aux répertoires, des distinctions fondamentales entre chorégraphies (il serait même plus juste de dire que ces distinctions entre des mouvements de base de la danse sont fondatrices de distinctions socio-spatiales).
L'ouvrage commence par un panorama historique qui nous rappelle la dynamique des mouvements et des rencontres de différentes populations, dynamique considérée à partir des pratiques musicales de la région du Rio Negro. Ainsi, l'auteur nous rappelle que la présence des missionnaires catholiques est ancienne, et que les pratiques musicales syncrétiques datent de plusieurs siècles. Nous aurions ainsi dans cette région une tradition particulière des mélanges musicaux. Il ne nous est pas toujours facile de nous retrouver dans les datations de l'époque coloniale, mais cette tentative pour une esquisse historique de la présence des missionnaires catholiques réussit à nous montrer la multiplicité des influences missionnaires ellesmêmes. C'est un thème important, une contribution à la critique des modèles opposant deux ensembles qui seraient homogènes: l'histoire indigène d'un côté, et l'occupation missionnaire de l'autre.

Puis, le corps de l'ethnographie commence par tout un chapitre entier consacré au Festribal. Le Festribal est une des ces rencontres culturelles, innombrables dans le monde, où à travers des présentation de musique, danse, sport, artisanat, cuisine, se mêlent affirmation identitaire, tourisme, folklorisation, revitalisation, commerce etc. Et il est bon que nous en soit offerte ici une description intime, dont certains éléments formulés par les acteurs eux-mêmes, posent des questions à portée plus générale. Cet évènement est une des principales occasions où les catégories culturelles se croisent, se réactivent et se transforment. La description des répétitions de certains groupes, le relevé des injonctions du Conseil municipal, informent sur les contenus et les intentions attribuées à cet évènement annuel. Cette ethnographie amène ainsi des questions sur les différentes stratégies culturelles: celle de la municipalité, celles des associations, celles des ruraux proches de la ville, celles des communautés du haut fleuve. Nous avons aussi un aperçu des oppositions et des négociations entre ces différents projets. Enfin, les spectacles et les réactions des spectateurs mettent en œuvre des phénomènes de réverbération qui critiquent de manière profonde les catégories locales, non locales et universitaires:

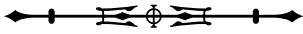


des 'índios', des 'descendants d'índios' jouent aux 'índios', les uns devant les autres (p. 99-100).

Le chapitre suivant est consacré aux classifications des répertoires musicaux. Les trois grandes catégories locales sont: repertórios culturais, de la região et de fora. L'intérêt de ce chapitre est qu'il tente de nous présenter ces répertoires de manière croisée: plusieurs classification coexistantes peuvent simultanément attribuer des places (des classes) différentes, voire conflictuelles à la même musique: il en est ainsi des classifications des communautés indigènes et de celle du Festribal. La description ethnomusicographique de ces musiques, tout en étant utile et globalement bien faite (les transcriptions sont de qualité), souffrent néanmoins de quelques imprécisions terminologiques.

Enfin, le dernier chapitre reprend ces classifications dans une perspective de définition identitaire. Nous y retrouvons une formulation à la fois juste et féconde: ce texte nous propose une ethnologie d'une 'culture de contact'. Là est à mon sens l'originalité du livre: c'est, à ma connaissance, la première étude portant sur les musiques de communautés indigènes urbaines au Brésil. Certains dispositifs et certaines dynamiques mises au jour ici se retrouvent dans d'autres régions d'Amérique du Sud. L'auteure nous rappelle aussi que cette étude s'est réalisée dans le cadre d'une recherche-action. Recherche militante et partagée qui, fort heureusement, est de plus en plus répandue grâce à la pression des communautés indigènes elles-mêmes.

L'ensemble de l'ouvrage est alimenté de nombreuses et longues citations, qui sont surtout intéressantes lorsque ce sont les musiciens, les acteurs des rencontres culturelles qui parlent (les citations d'ethnologues auraient méritées d'être plus synthétisées). Pour terminer, on doit féliciter l'éditeur pour la publication de cet ouvrage. En effet, au Brésil trop peu de livres sur ces musiques sont encore publiés, alors que l'ethnomusicologie y est actuellement en pleine expansion: multiplication des cursus universitaires ainsi que des mastères et des doctorats.

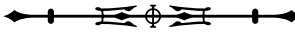

